\title{
Effects of Coinfection on the Dynamics of Two Pathogens in a Tick-Host Infection Model
}

\author{
Bei Sun, ${ }^{1}$ Xue Zhang $\mathbb{D}^{1},{ }^{1}$ and Marco Tosato $^{2}$ \\ ${ }^{1}$ College of Science, Northeastern University, Shenyang, Liaoning 110819, China \\ ${ }^{2}$ Laboratory for Industrial and Applied Mathematics, York University, Toronto, Ontario M3J1P3, Canada \\ Correspondence should be addressed to Xue Zhang; zhangxue@mail.neu.edu.cn
}

Received 12 November 2019; Accepted 1 April 2020; Published 28 May 2020

Academic Editor: Xinzhi Liu

Copyright ( $\odot 2020$ Bei Sun et al. This is an open access article distributed under the Creative Commons Attribution License, which permits unrestricted use, distribution, and reproduction in any medium, provided the original work is properly cited.

As both ticks and hosts may carry one or more pathogens, the phenomenon of coinfection of multiple tick-borne diseases becomes highly relevant and plays a key role in tick-borne disease transmission. In this paper, we propose a coinfection model involving two tickborne diseases in a tick-host population and calculate the basic reproduction numbers at the disease-free equilibrium and two boundary equilibria. To explore the impact of coinfection, we also derive the invasion reproduction numbers which indicate the potential of a pathogen to persist when another pathogen already exists in tick and host populations. Then, we obtain the global stability of the system at the disease-free equilibrium and the boundary equilibrium, respectively, and further demonstrate the existence conditions for uniform persistence of the two diseases. The final numerical simulations mainly verify the theoretical results of coinfection.

\section{Introduction}

Tick-borne diseases, which mainly comprise Lyme disease, human babesiosis, tick-borne encephalitis, and human granulocytic, are becoming an increasingly significant danger to people living in countrysides or near woodlands all over the world. Specifically, Lyme disease, which is one of the most widespread tick-born diseases in the world, is caused by the pathogen Borrelia burgdorferi, and medical reports prove that there were more than 20,000 cases already as early as 2013 in America [1] and that the number has continued to increase year after year. As for human babesiosis, the parasite Babesia microti is responsible for the occurrence of this disease, and the number of human cases is in steady increase in northeastern America [2]. Tick-borne encephalitis, instead, is an arthropod-transmitted viral infection caused by TBE virus and frequently occurs in some European countries. Human granulocytic anaplasmosis is caused by Anaplasma phagocytophilum that is a bacterium, which has done great harm to human health over the last decade [3].

The main species that transmit all the tick-borne diseases mentioned above are Ixodes ticks, so their dynamics plays a significant role in studying the transmission of tick-borne diseases. For instance, a black-legged tick named Ixodes scapularis can be infected by a large number of different kinds of pathogens including $B$. burgdorferi, B. microti, and $A$. phagocytophilum or any combination of them concurrently [4]. An early study reported occurrences of coinfection of different pathogens in tick and host populations [5], and several case studies described the coinfection among tick-borne diseases from biotic experiments and ecological perspectives.

Diuk-Wasser et al. [6] analyzed the promoting effect of coinfection of B. burgdorferi and B. microti on disease transmission in conformity with epidemiological, ecological, and clinical effects. Horowitz et al. [7] applied blood culture and serology to detect how human granulocytic anaplasmosis in Lyme disease infections played a role in the apparent rate of coinfection and in the severity to illness by studying concrete cases to explore their interaction. Welc-Faleciak et al. [8] performed a retrospective study concerning tick-exposed hosts living in southeastern Poland, aimed at probing into the risk of coinfection between Borrelia species and Anaplasma phagocytophilum or Babesia spp.. These articles have 
clearly shown that coinfection among tick-borne pathogens or diseases is a real phenomenon through actual case studies or experimental analysis and demonstrated that coinfection can indeed have a certain impact on the disease transmission.

Mathematical models have been applied to the coinfection of infectious diseases. Gao et al. [9] established a SIS epidemic model that includes coinfection between two infectious diseases and proved a sufficient condition for coexistence of both diseases by introducing the invasion reproduction number. Tang et al. [10] integrated the relevant transmission dynamics describing coinfection of dengue and Zika into a mathematical model and focused on how dengue vaccine affected the outbreak of Zika. Wang et al. [11] proposed a Zika-dengue model emphasizing the joint dynamics between dengue and Zika and discussed the impact of vaccination against dengue and antibody-development enhancement. These studies are all related to mosquito-borne diseases; however, to our best knowledge, reports of mathematical models regarding tick-borne coinfection are relatively scarce. Exclusively, Lou et al. [2] built a tick-borne pathogen model with coinfection and discussed the promotion effect of coinfection on two diseases transmission. Unfortunately, they just considered coinfection with two determinated diseases, Lyme disease and human babesiosis, and ignored the effects of invasion on coexistence of the two pathogens.

Based on the previous studies mentioned above, our objective here is principally to formulate a tick-borne coinfection model comprising tick dynamics and host dynamics with two pathogens and obtain the coexistence conditions of two tick-borne diseases. This paper is organized as follows. We establish a coinfection model involving tick and host populations in Section 2. Then, we calculate basic reproduction numbers and invasion reproduction numbers and present their explicit expressions in Section 3. We further discuss the threshold dynamics at the disease- free equilibrium and two boundary equilibria, as well as the coexistence conditions of two tick-borne diseases in Section 4. Numerical simulations validate the coinfection theories and reveal the coexistence conditions of two diseases, and finally, we provide some discussions in Section 5.

\section{The Model}

We formulate a SIS-type model consisting of two tick-borne pathogens to describe the disease dynamics of coinfection as well as the effect on the spread of two tick-borne diseases. Note that we only consider one life stage of tick, nymph, which can account for the infection on the host to a great extent [12]. Both ticks and hosts can be infected with two or more pathogens through tick bites and blood meals.

Let $T(t)$ and $H(t)$ be the total tick population and host population, respectively. The tick population is divided into four subclasses: susceptible ticks to two diseases, infected ticks with only disease 1 , infected ticks with only disease 2 , and infected ticks with both diseases, which can be described as $T^{s}(t), T_{1}^{i}(t), T_{2}^{i}(t)$, and $T_{3}^{i}(t)$, respectively. Here, the total tick population is

$$
T(t)=T^{s}(t)+T_{1}^{i}(t)+T_{2}^{i}(t)+T_{3}^{i}(t) .
$$

The host population is also partitioned into four categories: susceptible hosts to two diseases, infected hosts with only disease 1 , infected hosts with only disease 2 , and infected hosts with both diseases, which are expressed as $H^{s}(t), H_{1}^{i}(t)$, $H_{2}^{i}(t)$, and $H_{3}^{i}(t)$, respectively. In the same way,

$$
H(t)=H^{s}(t)+H_{1}^{i}(t)+H_{2}^{i}(t)+H_{3}^{i}(t)
$$

In combination with the above statements, a transmission diagram describing coinfection of two diseases transmission among ticks and hosts is explicitly depicted in Figure 1. Then, we construct the following model:

$$
\begin{aligned}
& \frac{d T^{s}(t)}{d t}=\Lambda_{T}-\left(\beta_{1} H_{1}^{i}(t)+\beta_{2} H_{2}^{i}(t)+\beta_{3} H_{3}^{i}(t)+\beta_{31} H_{3}^{i}(t)+\beta_{32} H_{3}^{i}(t)\right) \frac{T^{s}(t)}{H(t)}-\mu_{T} T^{s}(t) \\
& \frac{d T_{1}^{i}(t)}{d t}=\beta_{1} H_{1}^{i}(t) \frac{T^{s}(t)}{H(t)}+\beta_{31} H_{3}^{i}(t) \frac{T^{s}(t)}{H(t)}-\beta_{12} H_{2}^{i}(t) \frac{T_{1}^{i}(t)}{H(t)}-\beta_{13} H_{3}^{i}(t) \frac{T_{1}^{i}(t)}{H(t)}-\mu_{T} T_{1}^{i}(t) \\
& \frac{d T_{2}^{i}(t)}{d t}=\beta_{2} H_{2}^{i}(t) \frac{T^{s}(t)}{H(t)}+\beta_{32} H_{3}^{i}(t) \frac{T^{s}(t)}{H(t)}-\beta_{21} H_{1}^{i}(t) \frac{T_{2}^{i}(t)}{H(t)}-\beta_{23} H_{3}^{i}(t) \frac{T_{2}^{i}(t)}{H(t)}-\mu_{T} T_{2}^{i}(t) \\
& \frac{d T_{3}^{i}(t)}{d t}=\beta_{3} H_{3}^{i}(t) \frac{T^{s}(t)}{H(t)}+\beta_{12} H_{2}^{i}(t) \frac{T_{1}^{i}(t)}{H(t)}+\beta_{13} H_{3}^{i}(t) \frac{T_{1}^{i}(t)}{H(t)}+\beta_{21} H_{1}^{i}(t) \frac{T_{2}^{i}(t)}{H(t)}+\beta_{23} H_{3}^{i}(t) \frac{T_{2}^{i}(t)}{H(t)}-\mu_{T} T_{3}^{i}(t) \\
& \frac{d H^{s}(t)}{d t}=\Lambda_{H}-\left(\alpha_{1} T_{1}^{i}(t)+\alpha_{2} T_{2}^{i}(t)+\alpha_{3} T_{3}^{i}(t)+\alpha_{31} T_{3}^{i}(t)+\alpha_{32} T_{3}^{i}(t)\right) \frac{H^{s}(t)}{H(t)}-\mu_{H} H^{s}(t) \\
& \frac{d H_{1}^{i}(t)}{d t}=\alpha_{1} T_{1}^{i}(t) \frac{H^{s}(t)}{H(t)}+\alpha_{31} T_{3}^{i}(t) \frac{H^{s}(t)}{H(t)}-\alpha_{12} T_{2}^{i}(t) \frac{H_{1}^{i}(t)}{H(t)}-\alpha_{13} T_{3}^{i}(t) \frac{H_{1}^{i}(t)}{H(t)}-\mu_{H} H_{1}^{i}(t) \\
& \frac{d H_{2}^{i}(t)}{d t}=\alpha_{2} T_{2}^{i}(t) \frac{H^{s}(t)}{H(t)}+\alpha_{32} T_{3}^{i}(t) \frac{H^{s}(t)}{H(t)}-\alpha_{21} T_{1}^{i}(t) \frac{H_{2}^{i}(t)}{H(t)}-\alpha_{23} T_{3}^{i}(t) \frac{H_{2}^{i}(t)}{H(t)}-\mu_{H} H_{2}^{i}(t) \\
& \frac{d H_{3}^{i}(t)}{d t}=\alpha_{3} T_{3}^{i}(t) \frac{H^{s}(t)}{H(t)}+\alpha_{12} T_{2}^{i}(t) \frac{H_{1}^{i}(t)}{H(t)}+\alpha_{13} T_{3}^{i}(t) \frac{H_{1}^{i}(t)}{H(t)}+\alpha_{21} T_{1}^{i}(t) \frac{H_{2}^{i}(t)}{H(t)}+\alpha_{23} T_{3}^{i}(t) \frac{H_{2}^{i}(t)}{H(t)}-\mu_{H} H_{3}^{i}(t)
\end{aligned}
$$




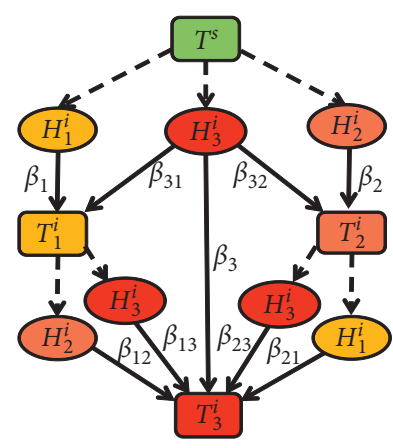

(a)

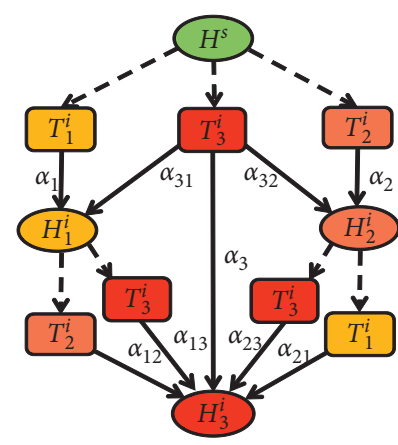

(b)

FIgURE 1: A transmission diagram of coinfection of two tick-borne pathogens among tick population (a) and host population (b), respectively.

where all parameters involved in the model are listed in Table 1. We assume the summations of transmission probabilities $\beta_{1}+\beta_{31}$ and $\alpha_{1}+\alpha_{31}$ are greater than original $\beta_{1}$ and $\alpha_{1}$ without coinfection, respectively, and which also hold for the transmission probabilities of disease 2. Susceptible ticks $T^{s}(t)$ recruited at a constant rate $\Lambda_{T}$ are infected and can move into $T_{1}^{i}(t)\left(T_{2}^{i}(t)\right)$ by blood feeding on $H_{1}^{i}(t)$ or $H_{3}^{i}(t)$ $\left(H_{2}^{i}(t)\right.$ or $\left.H_{3}^{i}(t)\right)$ with rates of disease transfer $\beta_{1}, \beta_{31} \geq 0\left(\beta_{2}\right.$, $\left.\beta_{32} \geq 0\right)$. $T_{1}^{i}(t) \quad\left(T_{2}^{i}(t)\right)$ can move to $T_{3}^{i}(t)$ by feeding on $H_{2}^{i}(t)$ or $H_{3}^{i}(t)\left(H_{1}^{i}(t)\right.$ or $\left.H_{3}^{i}(t)\right)$ with infection probability $\beta_{12}, \beta_{13} \geq 0\left(\beta_{21}, \beta_{23} \geq 0\right)$. In addition, susceptible ticks $T^{s}(t)$ may also be infected by taking a blood meal from $H_{3}^{i}(t)$ directly with infection probability $\beta_{3}$ and move into compartment $T_{3}^{i}(t)$. We also consider $\mu_{T}$ which represents the exit rate from the current compartment of the tick population. Similarly, the infection process with two diseases in the host population can be derived.

For the total populations of ticks and hosts, it can be shown that

$$
\begin{aligned}
& \frac{\mathrm{d} T(t)}{\mathrm{d} t}=\Lambda_{T}-\mu_{T} T(t), \\
& \frac{\mathrm{d} H(t)}{\mathrm{d} t}=\Lambda_{H}-\mu_{H} H(t) .
\end{aligned}
$$

It follows that the asymptotic equilibria of the two populations satisfy $\lim _{t \rightarrow+\infty} T(t)=\Lambda_{T} / \mu_{T}$ and $\lim _{t \rightarrow+\infty} H(t)=$ $\Lambda_{H} / \mu_{H}$. Here, Lemma 1 illustrates the basic properties of solutions in system (3), and the proof corresponding to this lemma can be derived in the Appendix of Wang [11].

Lemma 1. Let $\chi(t)=\left(T^{s}(t), T_{1}^{i}(t), T_{2}^{i}(t), T_{3}^{i}(t), H^{s}(t), H_{1}^{i}\right.$ $\left.(t), H_{2}^{i}(t), H_{3}^{i}(t)\right)$. All solutions $\chi(t)$ of system (3) with nonnegative initial values $\chi(0)$ remain nonnegative for $t \geq 0$. Specifically, the solutions $\chi(t)$ satisfy $\chi(t)>0$ under the condition that the initial value $\chi(0)>0$ for $t>0$. Furthermore, the solution set $\Gamma=\left\{\left(T^{s}(t), T_{1}^{i}(t), T_{2}^{i}(t), T_{3}^{i}(t), H^{s}\right.\right.$ $\left.(t), H_{1}^{i}(t), H_{2}^{i}(t), H_{3}^{i}(t)\right) \mid\left(T^{s}(t) \geq 0, T_{1}^{i}(t) \geq 0, T_{2}^{i}(t) \geq 0, T_{3}^{i}\right.$ $\left.(t) \geq 0, H^{s}(t) \geq 0, H_{1}^{i}(t) \geq 0, H_{2}^{i}(t) \geq 0, H_{3}^{i}(t)\right) \geq 0,0 \leq T^{s}(t)+$ $T_{1}^{i}(t)+T_{2}^{i}(t)+T_{3}^{i}(t) \leq \Lambda_{T} / \mu_{T}, 0 \leq H^{s}(t)+H_{1}^{i}(t)+H_{2}^{i}(t)$ $\left.+H_{3}^{i}(t) \leq \Lambda_{H} / \mu_{H}\right\}$ is positively invariant.

\section{The Reproduction Numbers}

The reproduction number $\mathscr{R}_{0}$, which is an essential threshold concept in epidemiological studies, denotes the average number of secondary cases caused by an infectious individual when it is introduced in an entirely susceptible population [11]. Mathematically, $\mathscr{R}_{0}$ can measure the maximum reproductive capacity and determine whether the disease will become endemic or die out [13]. In this section, we calculate basic reproduction numbers and invasion reproduction numbers.

3.1. Basic Reproduction Number. In system (3), there always exists a disease-free equilibrium $E_{0}=\left(T_{0}^{s}, 0,0,0, H_{0}^{s}, 0,0,0\right)$ in which $T_{0}^{s}=\Lambda_{T} / \mu_{T}$ and $H_{0}^{s}=\Lambda_{H} / \mu_{H}$. Based on the calculation of the relevant next generation matrices [14], we can easily obtain the specific form of the basic reproduction number, that is,

$$
\mathscr{R}_{0}=\max \left\{\mathscr{R}_{1}, \mathscr{R}_{2}, \mathscr{R}_{3}\right\},
$$

where $\mathscr{R}_{i}=\sqrt{\left(\alpha_{i} \beta_{i} \Lambda_{T} / \Lambda_{H} \mu_{T}^{2}\right)}$ for $i=1,2,3$. Biologically, $\mathscr{R}_{1}$ indicates the average number of secondary cases infected by an infectious tick or host with disease 1 when this infection is drawn into a susceptible population. Concretely, it is the product of the number of new ticks infected with disease 1 , which are those produced by susceptible ticks feeding on an infectious host with disease 1 at a rate $\beta_{1} T_{0}^{s} / H$ during this host's average lifetime $1 / \mu_{H}$ and the number of new hosts infected with disease 1 generated by an infectious tick with disease 1 when taking blood meals of susceptible hosts at a rate $\alpha_{1} H_{0}^{s} / H$ during the period $1 / \mu_{T}$. The biological interpretations for $\mathscr{R}_{2}$ and $\mathscr{R}_{3}$ are analogous.

It is clear that there are two boundary equilibria $E_{1}$ and $E_{2}$, which represent the cases in which just disease 1 and just disease 2 are endemic in the population, respectively.

Therefore, at $E_{1}$, we have that $T_{2}^{i}, T_{3}^{i}, H_{2}^{i}$, and $H_{3}^{i}$ are all zero, and

$$
E_{1}=\left(T^{s *}, T_{1}^{i *}, 0,0, H^{s *}, H_{1}^{i *}, 0,0\right)
$$

where 
TABle 1: Model parameters and descriptions.

\begin{tabular}{|c|c|}
\hline Parameter & Description \\
\hline$\Lambda_{T}$ & Recruitment rate of ticks \\
\hline$\Lambda_{H}$ & Recruitment rate of hosts \\
\hline$\mu_{T}$ & Exit rate of ticks \\
\hline$\mu_{H}$ & Mortality rate of hosts \\
\hline$\beta_{1}, \beta_{2}$ & Probability of infection for susceptible ticks that feed on infectious hosts $H_{1}^{i}$ or $H_{2}^{i}$, respectively \\
\hline$\beta_{3}$ & Probability of infection for susceptible ticks that feed on infectious hosts $H_{3}^{i}$ and become infected by both pathogens \\
\hline$\beta_{31}, \beta_{32}$ & $\begin{array}{c}\text { Probability of infection for susceptible ticks that feed on infectious hosts } H_{3}^{i} \text { and become infected by only disease } 1 \text { or only } \\
\text { disease } 2 \text {, respectively }\end{array}$ \\
\hline$\beta_{12}, \beta_{13}$ & $\begin{array}{c}\text { Probability of infection for infectious ticks with only disease } 1 \text { that feed on } H_{2}^{i} \text { or } H_{3}^{i} \text {, respectively, and also become infected by } \\
\text { disease } 2\end{array}$ \\
\hline$\beta_{21}, \beta_{23}$ & $\begin{array}{c}\text { Probability of infection for infectious ticks with only disease } 2 \text { that feed on } H_{1}^{i} \text { or } H_{3}^{i} \text {, respectively, and also become infected by } \\
\text { disease } 1\end{array}$ \\
\hline$\alpha_{1}, \alpha_{2}$ & Probability of infection for susceptible hosts that are fed by infectious ticks $T_{1}^{i}$ or $T_{2}^{i}$, respectively. \\
\hline$\alpha_{3}$ & Probability of infection for susceptible hosts that are fed by infectious ticks $T_{3}^{i}$ and become infected by both pathogens \\
\hline$\alpha_{31}, \alpha_{32}$ & $\begin{array}{c}\text { Probability of infection for susceptible hosts that are fed by infectious ticks } T_{3}^{i} \text { and become infected by only disease } 1 \text { or only } \\
\text { disease } 2 \text {, respectively }\end{array}$ \\
\hline$\alpha_{12}, \alpha_{13}$ & $\begin{array}{c}\text { Probability of infection for infectious hosts with only disease } 1 \text { that are fed by } T_{2}^{i} \text { or } T_{3}^{i} \text {, respectively, and also become infected } \\
\text { by disease } 2\end{array}$ \\
\hline$\alpha_{21}, \alpha_{23}$ & $\begin{array}{c}\text { Probability of infection for infectious hosts with only disease } 2 \text { that are fed by } T_{1}^{i} \text { or } T_{3}^{i} \text {, respectively, and also become infected } \\
\text { by disease } 1\end{array}$ \\
\hline
\end{tabular}

$$
\begin{aligned}
T^{s *} & =\frac{\Lambda_{T}}{\mu_{T}+\lambda_{T}^{*}}, \\
T_{1}^{i *} & =\frac{\lambda_{T}^{*} \Lambda_{T}}{\mu_{T}\left(\mu_{T}+\lambda_{T}^{*}\right)}, \\
H^{s *} & =\frac{\Lambda_{H}}{\mu_{H}+\lambda_{H}^{*}}, \\
H_{1}^{i *} & =\frac{\lambda_{H}^{*} \Lambda_{H}}{\mu_{H}\left(\mu_{H}+\lambda_{H}^{*}\right)},
\end{aligned}
$$

in which

$$
\begin{aligned}
& \lambda_{T}^{*}=\frac{\beta_{1} H_{1}^{i *}}{H^{*}}, \\
& \lambda_{H}^{*}=\frac{\alpha_{1} T_{1}^{i *}}{H^{*}},
\end{aligned}
$$

with $H^{*}=H^{s *}+H_{1}^{i *}$.

We substitute (7) into (8) and get

$$
\lambda_{H}^{*}=\frac{\alpha_{1} \lambda_{T}^{*} \Lambda_{T}}{\mu_{T}\left(\mu_{T}+\lambda_{T}^{*}\right) H^{*}} .
$$

Moreover, there also exists

$$
\lambda_{H}^{*}=\frac{\lambda_{T}^{*} \mu_{H}^{2} H^{*}}{\beta_{1} \Lambda_{H}-\lambda_{T}^{*} \mu_{H} H^{*}} .
$$

Combining (9) and (10), we obtain

$$
\lambda_{T}^{*}=\frac{\mu_{H} \mu_{T}^{2} H^{*}\left(\mathscr{R}_{1}^{2}-1\right)}{\alpha_{1} \Lambda_{T}+\mu_{T} \mu_{H} H^{*}} .
$$

Therefore, (11) is positive if $\mathscr{R}_{1}>1$, but when $\mathscr{R}_{1}<1$, no positive root can be found. Thus, the equilibrium $E_{1}$ exists provided $\mathscr{R}_{1}>1$.

Similarly, the boundary equilibrium $E_{2}$ takes the following form:

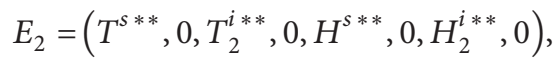

where

$$
\begin{aligned}
T^{\mathcal{*}^{* *}} & =\frac{\Lambda_{T}}{\mu_{T}+\lambda_{T}^{* *}}, \\
T_{2}^{i * *} & =\frac{\lambda_{T}^{* *} \Lambda_{T}}{\mu_{T}\left(\mu_{T}+\lambda_{T}^{* *}\right)}, \\
H^{s * *} & =\frac{\Lambda_{H}}{\mu_{H}+\lambda_{H}^{* *}}, \\
H_{2}^{i * *} & =\frac{\lambda_{H}^{* *} \Lambda_{H}}{\mu_{H}\left(\mu_{H}+\lambda_{H}^{* *}\right)},
\end{aligned}
$$

in which

$$
\begin{aligned}
& \lambda_{T}^{* *}=\frac{\beta_{2} H_{2}^{i * *}}{H^{* *}}, \\
& \lambda_{H}^{* *}=\frac{\alpha_{2} T_{2}^{i * *}}{H^{* *}},
\end{aligned}
$$

with $H^{* *}=H^{s *}+H_{2}^{i * *}$. Also, the equilibrium $E_{2}$ exists if $\mathscr{R}_{2}>1$.

3.2. Invasion Reproduction Number. The basic reproduction number works as a threshold that only includes information about diseases transmission in one population containing a single pathogen and focuses on the static state, but it cannot represent the number of secondary cases infected by an infectious individual with one disease when this infection is led into a population where another disease already exists. 
Thus, it is necessary to introduce a new threshold quantity: the invasion reproduction number [15].

In the following, we calculate two reproduction numbers which are the invasion reproduction number for disease 1 when disease 2 is already endemic and the invasion reproduction number for disease 2 when disease 1 is already endemic, respectively.

Considering that the derivation of the invasion reproduction number for disease 2, namely, $\mathscr{R}_{2}^{1}$, is based on a population in which disease 1 is already endemic, we concentrate on the boundary equilibrium $E_{1}$ to reckon the matrices $\mathscr{F}_{2}$ and $\mathscr{V}_{2}$ as follows:

$$
\left.\begin{array}{c}
\mathscr{F}_{2}\left[\begin{array}{c}
\frac{\beta_{2} H_{2}^{i} T^{s *}}{H^{*}+H_{2}^{i}+H_{3}^{i}}+\frac{\beta_{32} H_{3}^{i} T^{s *}}{H^{*}+H_{2}^{i}+H_{3}^{i}} \\
\frac{\beta_{3} H_{3}^{i} T^{s *}}{H^{*}+H_{2}^{i}+H_{3}^{i}}+\frac{\beta_{12} H_{2}^{i} T_{1}^{i *}}{H^{*}+H_{2}^{i}+H_{3}^{i}}+\frac{\beta_{13} H_{3}^{i} T_{1}^{i *}}{H^{*}+H_{2}^{i}+H_{3}^{i}} \\
\frac{\alpha_{2} T_{2}^{i} H^{s *}}{H^{*}+H_{2}^{i}+H_{3}^{i}}+\frac{\alpha_{32} T_{3}^{i} H^{s *}}{H^{*}+H_{2}^{i}+H_{3}^{i}} \\
\frac{\alpha_{3} T_{3}^{i} H^{s *}}{H^{*}+H_{2}^{i}+H_{3}^{i}}+\frac{\alpha_{12} T_{2}^{i} H_{1}^{i *}}{H^{*}+H_{2}^{i}+H_{3}^{i}}+\frac{\alpha_{13} T_{3}^{i} H_{1}^{i *}}{H^{*}+H_{2}^{i}+H_{3}^{i}}
\end{array}\right] \\
\mu_{T} T_{2}^{i}+\frac{\beta_{21} T_{2}^{i} H_{1}^{i *}}{H^{*}+H_{2}^{i}+H_{3}^{i}}+\frac{\beta_{23} H_{3}^{i} T_{2}^{i}}{H^{*}+H_{2}^{i}+H_{3}^{i}} \\
\mathscr{V}_{T} T_{3}^{i}-\frac{\beta_{21} T_{2}^{i} H_{1}^{i *}}{H^{*}+H_{2}^{i}+H_{3}^{i}}-\frac{\beta_{23} H_{3}^{i} T_{2}^{i}}{H^{*}+H_{2}^{i}+H_{3}^{i}} \\
\mu_{H} H_{2}^{i}+\frac{\alpha_{21} H_{2}^{i} T_{1}^{i *}}{H^{*}+H_{2}^{i}+H_{3}^{i}}+\frac{\alpha_{23} T_{3}^{i} H_{2}^{i}}{H^{*}+H_{2}^{i}+H_{3}^{i}} \\
\mu_{H} H_{3}^{i}-\frac{\alpha_{21} H_{2}^{i} T_{1}^{i *}}{H^{*}+H_{2}^{i}+H_{3}^{i}}-\frac{\alpha_{23} T_{3}^{i} H_{2}^{i}}{H^{*}+H_{2}^{i}+H_{3}^{i}}
\end{array}\right] .
$$

Next, taking the derivative of $\mathscr{F}_{2}$ and $\mathscr{V}_{2}$ with respect to $\left(T_{2}^{i}, T_{3}^{i}, H_{2}^{i}, H_{3}^{i}\right)^{T}$ and substituting the boundary equilibrium $E_{1}$ into the variables, we have

$$
F_{2}=\left[\begin{array}{cccc}
0 & 0 & \frac{\beta_{2} T^{s *}}{H^{*}} & \frac{\beta_{32} T^{s *}}{H^{*}} \\
0 & 0 & \frac{\beta_{12} T_{1}^{i *}}{H^{*}} \frac{\beta_{3} T^{s *}+\beta_{13} T_{1}^{i *}}{H^{*}} \\
\frac{\alpha_{2} H^{s *}}{H^{*}} & \frac{\alpha_{32} H^{s *}}{H^{*}} & 0 & 0 \\
\frac{\alpha_{12} H_{1}^{i *}}{H^{*}} \frac{\alpha_{3} H^{s *}+\alpha_{13} H_{1}^{i *}}{H^{*}} & 0 & 0
\end{array}\right],
$$

$$
V_{2}=\left[\begin{array}{cccc}
\mu_{T}+\frac{\beta_{21} H_{1}^{i *}}{H^{*}} & 0 & 0 & 0 \\
-\frac{\beta_{21} H_{1}^{i *}}{H^{*}} & \mu_{T} & 0 & 0 \\
0 & 0 & \mu_{H}+\frac{\alpha_{21} T_{1}^{i *}}{H^{*}} & 0 \\
0 & 0 & -\frac{\alpha_{21} T_{1}^{i *}}{H^{*}} & \mu_{H}
\end{array}\right] .
$$

Then, the next generation matrix is given by

$$
F_{2} V_{2}^{-1}=\left[\begin{array}{llll}
0 & 0 & a & b \\
0 & 0 & c & d \\
e & f & 0 & 0 \\
k & g & 0 & 0
\end{array}\right],
$$

where

$$
\begin{aligned}
& a=\frac{\mu_{H} H^{*} \beta_{2} T^{s *}+\alpha_{21} T_{1}^{i *} \beta_{32} T^{s *}}{\mu_{H} H^{*}\left(\mu_{H} H^{*}+\alpha_{21} T_{1}^{i *}\right)}, \\
& b=\frac{\beta_{32} T^{s *}}{\mu_{H} H^{*}}, \\
& c=\frac{\beta_{12} T_{1}^{i *} \mu_{H} H^{*}+\alpha_{21} T_{1}^{i *} \beta_{3} T^{s *}+\beta_{13} T_{1}^{i *} \alpha_{21} T_{1}^{i *}}{\mu_{H} H^{*}\left(\mu_{H} H^{*}+\alpha_{21} T_{1}^{i *}\right)}, \\
& d=\frac{\beta_{3} T^{s *}+\beta_{13} T_{1}^{i *}}{\mu_{H} H^{*}}, \\
& e=\frac{\alpha_{2} H^{s *} \mu_{T} H^{*}+\alpha_{32} H^{s *} \beta_{21} H_{1}^{i *}}{\mu_{T} H^{*}\left(\mu_{T} H^{*}+\beta_{21} H_{1}^{* *}\right)}, \\
& f=\frac{\alpha_{32} H^{s *}}{\mu_{T} H^{*}}, \\
& k=\frac{\alpha_{12} H_{1}^{i *} \mu_{T} H^{*}+\alpha_{3} H^{s *} \beta_{21} H_{1}^{i *}+\alpha_{13} H_{1}^{i *} \beta_{21} H_{1}^{i *}}{\mu_{T} H^{*}\left(\mu_{T} H^{*}+\beta_{21} H_{1}^{i *}\right)}, \\
& g=\frac{\alpha_{3} H^{s *}+\alpha_{13} H_{1}^{i *}}{\mu_{T} H^{*}},
\end{aligned}
$$

in which $T^{s *}, T_{1}^{i *}, H^{s *}, H_{1}^{i *}$, and $H^{*}$ are expressed in (7). The characteristic equation is

$$
f(\lambda)=\lambda^{4}-(c f+a e+d g+b k) \lambda^{2}+(e g-k f)(a d-b c)=0 .
$$

Let

$$
\begin{aligned}
& A=(c f+a e+d g+b k), \\
& B=(e g-k f)(a d-b c) .
\end{aligned}
$$

It follows from $A^{2}-4 B>0$ that the dominant eigenvalue of equation (19) satisfies $\lambda^{2}=\left(\left(A+\sqrt{A^{2}-4 B}\right) / 2\right)$, and then we can acquire the invasion reproduction number for disease 2, that is, $\left.\mathscr{R}_{2}^{1}=\rho\left(F_{2} V_{2}^{-1}\right)=\sqrt{\left(\left(A+\sqrt{A^{2}-4 B}\right) / 2\right.}\right)$. 
Similarly, the invasion reproduction number for disease 1, namely, $\mathscr{R}_{1}^{2}$, can also be derived following the above process, which is given by

$$
\mathscr{R}_{1}^{2}=\rho\left(F_{1} V_{1}^{-1}\right)=\sqrt{\frac{\widetilde{A}+\sqrt{\widetilde{A}^{2}-4 \widetilde{B}}}{2}},
$$

where

$$
\begin{aligned}
& \widetilde{A}=(\tilde{c} \tilde{f}+\tilde{a} \widetilde{e}+\tilde{d} \widetilde{g}+\tilde{b} \tilde{k}), \\
& \widetilde{B}=(\widetilde{e} \widetilde{g}-\tilde{k} \widetilde{f})(\widetilde{a} \widetilde{d}-\widetilde{b} \widetilde{c}), \\
& \widetilde{a}=\frac{\mu_{H} H^{* *} \beta_{1} T^{s * *}+\alpha_{12} T_{2}^{i * *} \beta_{31} T^{s * *}}{\mu_{H} H^{* *}\left(\mu_{H} H^{* *}+\alpha_{12} T_{2}^{i * *}\right)}, \\
& \tilde{b}=\frac{\beta_{31} T^{s * *}}{\mu_{H} H^{* *}} \text {, } \\
& \tilde{c}=\frac{\beta_{21} T_{2}^{i * *} \mu_{H} H^{* *}+\alpha_{12} T_{2}^{i * *} \beta_{3} T^{s * *}+\beta_{12} T_{2}^{i * *} \alpha_{23} T_{2}^{i * *}}{\mu_{H} H^{* *}\left(\mu_{H} H^{* *}+\alpha_{12} T_{2}^{i * *}\right)}, \\
& \widetilde{d}=\frac{\beta_{3} T^{s * *}+\beta_{23} T_{2}^{i * *}}{\mu_{H} H^{* *}}, \\
& \widetilde{e}=\frac{\alpha_{1} H^{s * *} \mu_{T} H^{* *}+\alpha_{31} H^{s * *} \beta_{12} H_{2}^{i * *}}{\mu_{T} H^{* *}\left(\mu_{T} H^{* *}+\beta_{12} H_{2}^{i * *}\right)}, \\
& \widetilde{f}=\frac{\alpha_{31} H^{s * *}}{\mu_{T} H^{* *}} \text {, } \\
& \widetilde{k}=\frac{\alpha_{21} H_{2}^{i * *} \mu_{T} H^{* *}+\alpha_{3} H^{s * *} \beta_{12} H_{2}^{i * *}+\alpha_{23} H_{2}^{i * *} \beta_{12} H_{2}^{i * *}}{\mu_{T} H^{* *}\left(\mu_{T} H^{* *}+\beta_{12} H_{2}^{i * *}\right)}, \\
& \tilde{g}=\frac{\alpha_{3} H^{s * *}+\alpha_{23} H_{2}^{i * *}}{\mu_{T} H^{* *}} \text {. }
\end{aligned}
$$

Note that $T^{s * *}, T_{2}^{i * *}, H^{s * *}, H_{2}^{i * *}$, and $H^{* *}$ are defined in (13).

\section{Stability and Coinfection Dynamics}

To study the dynamics of two diseases transmission, it is essential to explore the stability of system (3) by studying that of the disease-free equilibrium and the one-disease equilibria for two subsystems and yield dynamic conditions for a second disease invasion and coinfection.

4.1. Stability of Disease-Free Equilibrium. Firstly, we discuss the stability of the disease-free equilibrium $E_{0}=\left(T_{0}^{s}, 0,0,0\right.$, $\left.H_{0}^{s}, 0,0,0\right)$, where $T_{0}^{s}=\Lambda_{T} / \mu_{T}$ and $H_{0}^{s}=\Lambda_{H} / \mu_{H}$.

Theorem 1. The disease-free equilibrium $E_{0}$ for system (3) is globally asymptotically stable if $\mathscr{R}_{0}<1$ but unstable if $\mathscr{R}_{0}>1$.
Proof. As mentioned above, the tick and host populations satisfy the following two equations:

$$
\begin{gathered}
\frac{d T}{d t}=\Lambda_{T}-\mu_{T} T, \\
\Longrightarrow T_{0}^{s}=\frac{\Lambda_{T}}{\mu_{T}}, \\
\frac{d H}{d t}=\Lambda_{H}-\mu_{H} H, \\
\Longrightarrow H_{0}^{s}=\frac{\Lambda_{H}}{\mu_{H}},
\end{gathered}
$$

which means that system (3) is globally attractive for the two populations [16]. Then, there exists small enough $\epsilon>0$ and $t_{1}=$ $t(\epsilon)>0$ such that $T_{0}^{s}-\epsilon \leq T^{s} \leq T_{0}^{s}+\epsilon, H_{0}^{s}-\epsilon \leq H^{s} \leq H_{0}^{s}+\epsilon$, $0 \leq T_{1}^{i} \leq \epsilon, 0 \leq T_{2}^{i} \leq \epsilon, 0 \leq T_{3}^{i} \leq \epsilon, 0 \leq H_{1}^{i} \leq \epsilon, 0 \leq H_{2}^{i} \leq \epsilon$, and $0 \leq H_{3}^{i} \leq \epsilon$ for any $t \geq t_{1}$. So, for $t \geq t_{1}$, we can obtain

$$
\begin{aligned}
& \frac{d T_{1}^{i}(t)}{d t} \leq \beta_{1} \frac{T_{0}^{s}+\epsilon}{H_{0}^{s}-\epsilon} H_{1}^{i}(t)+\beta_{31} \frac{T_{0}^{s}+\epsilon}{H_{0}^{s}-\epsilon} H_{3}^{i}(t)-\mu_{T} T_{1}^{i}(t), \\
& \frac{d T_{2}^{i}(t)}{d t} \leq \beta_{2} \frac{T_{0}^{s}+\epsilon}{H_{0}^{s}-\epsilon} H_{2}^{i}(t)+\beta_{32} \frac{T_{0}^{s}+\epsilon}{H_{0}^{s}-\epsilon} H_{3}^{i}(t)-\mu_{T} T_{2}^{i}(t), \\
& \frac{d T_{3}^{i}(t)}{d t} \leq \beta_{3} \frac{T_{0}^{s}+\epsilon}{H_{0}^{s}-\epsilon} H_{3}^{i}(t)+\left(\beta_{12}+\beta_{13}+\beta_{21}+\beta_{23}\right) \frac{\epsilon^{2}}{H_{0}^{s}-\epsilon} \\
& -\mu_{T} T_{3}^{i}(t) \\
& \frac{d H_{1}^{i}(t)}{d t} \leq \alpha_{1} \frac{H_{0}^{s}+\epsilon}{H_{0}^{s}-\epsilon} T_{1}^{i}(t)+\alpha_{31} \frac{H_{0}^{s}+\epsilon}{H_{0}^{s}-\epsilon} T_{3}^{i}(t)-\mu_{H} H_{1}^{i}(t) \\
& \frac{d H_{2}^{i}(t)}{d t} \leq \alpha_{2} \frac{H_{0}^{s}+\epsilon}{H_{0}^{s}-\epsilon} T_{2}^{i}(t)+\alpha_{32} \frac{H_{0}^{s}+\epsilon}{H_{0}^{s}-\epsilon} T_{3}^{i}(t)-\mu_{H} H_{2}^{i}(t), \\
& \frac{d H_{3}^{i}(t)}{d t} \leq \alpha_{3} \frac{H_{0}^{s}+\epsilon}{H_{0}^{s}-\epsilon} T_{3}^{i}(t)+\left(\alpha_{12}+\alpha_{13}+\alpha_{21}+\alpha_{23}\right) \frac{\epsilon^{2}}{H_{0}^{s}-\epsilon} \\
& -\mu_{H} H_{3}^{i}(t)
\end{aligned}
$$

We consider the auxiliary linear system

$$
\frac{d x(t)}{d t}=M_{0}(\epsilon) x(t)
$$

where vector $x(t)=\left(x_{1}(t), x_{2}(t), x_{3}(t), x_{4}(t), x_{5}(t), x_{6}\right.$ $(t))^{T}$ and 


$$
M_{0}(\epsilon)=\left[\begin{array}{cccccc}
-\mu_{T} & 0 & 0 & \beta_{1} \frac{T_{0}^{s}+\epsilon}{H_{0}^{s}-\epsilon} & 0 & \beta_{31} \frac{T_{0}^{s}+\epsilon}{H_{0}^{s}-\epsilon} \\
0 & -\mu_{T} & 0 & 0 & \beta_{2} \frac{T_{0}^{s}+\epsilon}{H_{0}^{s}-\epsilon} & \beta_{32} \frac{T_{0}^{s}+\epsilon}{H_{0}^{s}-\epsilon} \\
0 & 0 & -\mu_{T} & 0 & 0 & \beta_{3} \frac{T_{0}^{s}+\epsilon}{H_{0}^{s}-\epsilon} \\
\alpha_{1} \frac{H_{0}^{s}+\epsilon}{H_{0}^{s}-\epsilon} & 0 & \alpha_{31} \frac{H_{0}^{s}+\epsilon}{H_{0}^{s}-\epsilon} & -\mu_{H} & 0 & 0 \\
0 & \alpha_{2} \frac{H_{0}^{s}+\epsilon}{H_{0}^{s}-\epsilon} & \alpha_{32} \frac{H_{0}^{s}+\epsilon}{H_{0}^{s}-\epsilon} & 0 & -\mu_{H} & 0 \\
0 & 0 & \alpha_{3} \frac{H_{0}^{s}+\epsilon}{H_{0}^{s}-\epsilon} & 0 & 0 & -\mu_{H}
\end{array}\right] .
$$

When $\mathscr{R}_{0}<1$, it follows from Theorem 2 in [14] that $s\left(M_{0}(0)\right)<0$, which means that $M_{0}(0)$ is stable. Thus, we have $\lim _{t \longrightarrow+\infty} x_{i}(t)=0$ for $i=1,2, \ldots, 6$. In accordance with the comparison principle, we have

$$
\begin{aligned}
& \lim _{t \rightarrow+\infty}\left(T_{1}^{i}(t), T_{2}^{i}(t), T_{3}^{i}(t), H_{1}^{i}(t), H_{2}^{i}(t), H_{3}^{i}(t)\right) \\
& \quad=(0,0,0,0,0,0),
\end{aligned}
$$

and by the theory of asymptotically semiflow in [17], we get

$$
\lim _{t \longrightarrow+\infty} T^{s}(t)=T_{0}^{s} \quad \text { and } \quad \lim _{t \longrightarrow+\infty} H^{s}(t)=H_{0}^{s} .
$$

This proves that $E_{0}$ is globally asymptotically stable when $\mathscr{R}_{0}<1$. Similarly, there is small enough $\tau>0$ and $t_{2}=$ $t(\tau)>0$ such that $T_{0}^{s}-\tau \leq T^{s} \leq T_{0}^{s}+\tau, H_{0}^{s}-\tau \leq H^{s} \leq H_{0}^{s}+\tau$, $0 \leq T_{1}^{i} \leq \tau, 0 \leq T_{2}^{i} \leq \tau, 0 \leq T_{3}^{i} \leq \tau, 0 \leq H_{1}^{i} \leq \tau, 0 \leq H_{2}^{i} \leq \tau$, and $0 \leq H_{3}^{i} \leq \tau$ for any $t \geq t_{2}$. Thus, for $t \geq t_{2}$, we also have

$$
\begin{aligned}
& \frac{d T_{1}^{i}(t)}{d t} \geq \beta_{1} \frac{T_{0}^{s}-\tau}{H_{0}^{s}+\tau} H_{1}^{i}(t)+\beta_{31} \frac{T_{0}^{s}-\tau}{H_{0}^{s}+\tau} H_{3}^{i}(t)-\left(\beta_{12}+\beta_{13}\right) \frac{\tau^{2}}{H_{0}^{s}+\tau}-\mu_{T} T_{1}^{i}(t), \\
& \frac{d T_{2}^{i}(t)}{d t} \geq \beta_{2} \frac{T_{0}^{s}-\tau}{H_{0}^{s}+\tau} H_{2}^{i}(t)+\beta_{32} \frac{T_{0}^{s}-\tau}{H_{0}^{s}+\tau} H_{3}^{i}(t)-\left(\beta_{21}+\beta_{23}\right) \frac{\tau^{2}}{H_{0}^{s}+\tau}-\mu_{T} T_{2}^{i}(t), \\
& \frac{d T_{3}^{i}(t)}{d t} \geq \beta_{3} \frac{T_{0}^{s}-\tau}{H_{0}^{s}+\tau} H_{3}^{i}(t)-\mu_{T} T_{3}^{i}(t), \\
& \frac{d H_{1}^{i}(t)}{d t} \geq \alpha_{1} \frac{H_{0}^{s}-\tau}{H_{0}^{s}+\tau} T_{1}^{i}(t)+\alpha_{31} \frac{H_{0}^{s}-\tau}{H_{0}^{s}+\tau} T_{3}^{i}(t)-\left(\alpha_{12}+\alpha_{13}\right) \frac{\tau^{2}}{H_{0}^{s}+\tau}-\mu_{H} H_{1}^{i}(t), \\
& \frac{d H_{2}^{i}(t)}{d t} \geq \alpha_{2} \frac{H_{0}^{s}-\tau}{H_{0}^{s}+\tau} T_{2}^{i}(t)+\alpha_{32} \frac{H_{0}^{s}-\tau}{H_{0}^{s}+\tau} T_{3}^{i}(t)-\left(\alpha_{21}+\alpha_{23}\right) \frac{\tau^{2}}{H_{0}^{s}+\tau}-\mu_{H} H_{2}^{i}(t), \\
& \frac{d H_{3}^{i}(t)}{d t} \geq \alpha_{3} \frac{H_{0}^{s}-\tau}{H_{0}^{s}+\tau} T_{3}^{i}(t)-\mu_{H} H_{3}^{i}(t) .
\end{aligned}
$$

And we can construct the auxiliary linear system

$$
\frac{d x(t)}{d t}=M_{0}^{\prime}(\tau) x(t)
$$

where 


$$
M_{0}^{\prime}(\tau)=\left[\begin{array}{cccccc}
-\mu_{T} & 0 & 0 & \beta_{1} \frac{T_{0}^{s}-\tau}{H_{0}^{s}+\tau} & 0 & \beta_{31} \frac{T_{0}^{s}-\tau}{H_{0}^{s}+\tau} \\
0 & -\mu_{T} & 0 & 0 & \beta_{2} \frac{T_{0}^{s}-\tau}{H_{0}^{s}+\tau} & \beta_{32} \frac{T_{0}^{s}-\tau}{H_{0}^{s}+\tau} \\
0 & 0 & -\mu_{T} & 0 & 0 & \beta_{3} \frac{T_{0}^{s}-\tau}{H_{0}^{s}+\tau} \\
\alpha_{1} \frac{H_{0}^{s}-\tau}{H_{0}^{s}+\tau} & 0 & \alpha_{31} \frac{H_{0}^{s}-\tau}{H_{0}^{s}+\tau} & -\mu_{H} & 0 & 0 \\
0 & \alpha_{2} \frac{H_{0}^{s}-\tau}{H_{0}^{s}+\tau} & \alpha_{32} \frac{H_{0}^{s}-\tau}{H_{0}^{s}+\tau} & 0 & -\mu_{H} & 0 \\
0 & 0 & \alpha_{3} \frac{H_{0}^{s}-\tau}{H_{0}^{s}+\tau} & 0 & 0 & -\mu_{H}
\end{array}\right] .
$$

It is shown obviously that $s\left(M_{0}^{\prime}(0)\right)>0$ if $\mathscr{R}_{0}>1$, which implies that $y_{i}(t) \longrightarrow+\infty$ when $t \longrightarrow+\infty$ for $i=1,2$ $, \ldots, 6$. Then, the disease-free equilibrium $E_{0}$ is unstable.

4.2. Stability of Boundary Equilibria. We first analyze the two subsystems containing only one disease prior to exploring the stability of the boundary equilibria $E_{1}$ and $E_{2}$.

The subsystem with only disease 1 is given by

$$
\begin{aligned}
& \frac{d T^{s}(t)}{d t}=\Lambda_{T}-\beta_{1} H_{1}^{i}(t) \frac{T^{s}(t)}{H(t)}-\mu_{T} T^{s}(t), \\
& \frac{d T_{1}^{i}(t)}{d t}=\beta_{1} H_{1}^{i}(t) \frac{T^{s}(t)}{H(t)}-\mu_{T} T_{1}^{i}(t), \\
& \frac{d H^{s}(t)}{d t}=\Lambda_{H}-\alpha_{1} T_{1}^{i}(t) \frac{H^{s}(t)}{H(t)}-\mu_{H} H^{s}(t), \\
& \frac{d H_{1}^{i}(t)}{d t}=\alpha_{1} T_{1}^{i}(t) \frac{H^{s}(t)}{H(t)}-\mu_{H} H_{1}^{i}(t) .
\end{aligned}
$$

The feasible region of this subsystem (33) is $\Gamma_{1}=$ $\left\{\left(T^{s}(t), T_{1}^{i}(t), H^{s}(t), H_{1}^{i}(t)\right) \mid T^{s}(t) \geq 0, T_{1}^{i}(t) \geq 0, H^{s}(t) \geq 0\right.$, $H_{1}^{i}(t) \geq 0,0 \leq T^{s}(t)+T_{1}^{i}(t) \leq \Lambda_{T} / \mu_{T}, 0 \leq H^{s}(t)+H_{1}^{i}(t) \leq$ $\left.\Lambda_{H} / \mu_{H}\right\}$ and is positively invariant.

There is a disease-free equilibrium $E_{0}^{1}=\left(T_{0}^{s}, 0, H_{0}^{s}, 0\right)$ in subsystem (33), in which $T_{0}^{s}=\Lambda_{T} / \mu_{T}$ and $H_{0}^{s}=\Lambda_{H} / \mu_{H}$. If $\mathscr{R}_{1}>1$, then a unique endemic equilibrium $E_{1}^{1}=\left(T^{s *}\right.$, $\left.T_{1}^{i *}, H^{s *}, H_{1}^{i *}\right)$, where $T^{s *}, T_{1}^{i *}, H^{s *}$, and $H_{1}^{i *}$ are expressed as (7), exists in subsystem (33). We aim to study the global dynamics of system (33) as follows.

Theorem 2. The following statements are valid:

(i) If $\mathscr{R}_{1}<1$, then subsystem (33) at $E_{0}^{1}$ is globally asymptotically stable in $\Gamma_{1}$ (ii) If $\mathscr{R}_{1}>1$, then there exists a unique positive solution $E_{1}^{1}$ that is globally asymptotically stable for subsystem (33) in $\Gamma_{1} \backslash E_{0}^{1}$

Proof. In subsystem (33), the tick and host populations satisfy

$$
\begin{aligned}
& \frac{d T}{d t}=\Lambda_{T}-\mu_{T} T, \\
& \frac{d H}{d t}=\Lambda_{H}-\mu_{H} H,
\end{aligned}
$$

which imply that the subsystem is globally attractive, and there is $E_{0}^{1}=\left(T_{0}^{s}, 0, H_{0}^{s}, 0\right)$, where $T_{0}^{s}=\Lambda_{T} / \mu_{T}$ and $H_{0}^{s}=$ $\Lambda_{H} / \mu_{H}$

If $\mathscr{R}_{1}<1$, there exists small enough $\zeta>0$ and $t_{3}=$ $t(\zeta)>0$, and when $t \geq t_{3}$, we take the limiting system for $T_{1}^{i}$ and $H_{1}^{i}$. Considering the auxiliary system

$$
\frac{d w(t)}{d t}=M_{1}(\zeta) w(t)
$$

with vector $w(t)=\left(w_{1}(t), w_{2}(t)\right)$ and matrix

$$
M_{1}(\zeta)=\left[\begin{array}{cc}
-\mu_{T} & \beta_{1} \frac{T_{0}^{s}+\zeta}{H_{0}^{s}-\zeta} \\
\alpha_{1} \frac{H_{0}^{s}+\zeta}{H_{0}^{s}-\zeta} & -\mu_{H}
\end{array}\right],
$$

we have $s\left(M_{1}(0)\right)<0$ if $\mathscr{R}_{1}<1$ and finally obtain that $E_{0}^{1}$ is globally asymptotically stable when $\mathscr{R}_{1}<1$ according to the comparison principle.

Similarly, statement (ii) can also be proved via taking the limiting system for $T_{1}^{i}$ and $H_{1}^{i}$, a detailed proof process similar to Lemma 2.3 in Lou and Zhao [18] and Theorem 3.2 in Gao et al. [19].

Analogously, the other subsystem containing only disease 2 can be written as follows: 


$$
\begin{aligned}
& \frac{d T^{s}(t)}{d t}=\Lambda_{T}-\beta_{2} H_{2}^{i}(t) \frac{T^{s}(t)}{H(t)}-\mu_{T} T^{s}(t), \\
& \frac{d T_{2}^{i}(t)}{d t}=\beta_{2} H_{2}^{i}(t) \frac{T^{s}(t)}{H(t)}-\mu_{T} T_{2}^{i}(t), \\
& \frac{d H^{s}(t)}{d t}=\Lambda_{H}-\alpha_{2} T_{2}^{i}(t) \frac{H^{s}(t)}{H(t)}-\mu_{H} H^{s}(t), \\
& \frac{d H_{2}^{i}(t)}{d t}=\alpha_{2} T_{2}^{i}(t) \frac{H^{s}(t)}{H(t)}-\mu_{H} H_{2}^{i}(t) .
\end{aligned}
$$

In view of the feasible region $\Gamma_{2}=\left\{\left(T^{s}(t), T_{2}^{i}(t)\right.\right.$, $\left.H^{s}(t), H_{2}^{i}(t)\right) \mid T^{s}(t) \geq 0, T_{2}^{i}(t) \geq 0, H^{s}(t) \geq 0, H_{2}^{i}(t) \geq 0,0 \leq$ $\left.T^{s}(t)+T_{2}^{i}(t) \leq \Lambda_{T} / \mu_{T}, 0 \leq H^{s}(t)+H_{2}^{i}(t) \leq \Lambda_{H} / \mu_{H}\right\}$, we can find that $\Gamma_{2}$ is positively invariant. In this subsystem (37), there exists a disease-free equilibrium $E_{0}^{2}=\left(T_{0}^{s}, 0, H_{0}^{s}, 0\right)$ in which $T_{0}^{s}=\Lambda_{T} / \mu_{T}, H_{0}^{s}=\Lambda_{H} / \mu_{H}$, and a unique nontrivial equilibrium $E_{2}^{2}=\left(T^{s * *}, T_{2}^{i * *}, H^{s * *}, H_{2}^{i * *}\right)$ in which $T^{s * *}$, $T_{2}^{i * *}, H^{* * *}$, and $H_{2}^{i * *}$ are expressed by (13). Then, we obtain the global behaviors at $E_{0}^{2}$ and $E_{2}^{2}$ for subsystem (37).

\section{Theorem 3. The following statements are valid:}

(i) If $\mathscr{R}_{2}<1$, then subsystem (37) at $E_{0}^{2}$ is globally asymptotically stable in $\Gamma_{2}$

(ii) If $\mathscr{R}_{2}>1$, then there exists a unique positive solution $E_{2}^{2}$ that is globally asymptotically stable for subsystem (37) in $\Gamma_{2} \backslash E_{0}^{2}$

Next, we discuss the relevant dynamics of general system (3) in $\Gamma$, which comprise not only these basic reproduction numbers describing the stability of the boundary equilibria $E_{1}$ and $E_{2}$ but also the invasion reproduction numbers explaining how a disease can invade a tick-host population where another disease is already prevalent.

Theorem 4. When $\mathscr{R}_{1}>1$, the boundary equilibrium $E_{1}$ is locally asymptotically stable for system (3) if $\mathscr{R}_{2}^{1}<1$ but unstable if $\mathscr{R}_{2}^{1}>1$.

Proof. Define $W^{S}\left(E_{1}\right)$ as the stable manifold of $E_{1}$, and

$$
\begin{aligned}
\Gamma_{3}= & \left\{\left(T^{s}, T_{1}^{i}, T_{2}^{i}, T_{3}^{i}, H^{s}, H_{1}^{i}, H_{2}^{i}, H_{3}^{i}\right)\right. \\
& \left.\in \Gamma \mid T_{2}^{i}=T_{3}^{i}=H_{2}^{i}=H_{3}^{i}=0\right\}, \\
\Gamma_{4}= & \left\{\left(T^{s}, T_{1}^{i}, T_{2}^{i}, T_{3}^{i}, H^{s}, H_{1}^{i}, H_{2}^{i}, H_{3}^{i}\right)\right. \\
& \left.\in \Gamma \mid T^{s}=T^{s *}, T_{1}^{i}=T_{1}^{i *}, H^{s}=H^{s *}, H_{1}^{i}=H_{1}^{i *}\right\} .
\end{aligned}
$$

Note that $\Gamma_{3}$ and $\Gamma_{4}$ are all invariant sets for system (3) with respect to the boundary equilibrium $E_{1}$. According to the center manifold theorem, we consider two invariant manifolds $W_{1}$ and $W_{2}$ tangent to $\Gamma_{3}$ and $\Gamma_{4}$, respectively, which satisfy $\operatorname{dim} W_{1}=\operatorname{dim} \Gamma_{3}$ and $\operatorname{dim} W_{2}=\operatorname{dim} \Gamma_{4}$.

Likewise, $\Gamma_{3} \backslash E_{0}$ is also an invariant set for system (3), and there is an invariant manifold expressed as $W_{3}$ such that $\operatorname{dim} W_{3}=\operatorname{dim} \Gamma_{3} \backslash E_{0}=\operatorname{dim} \Gamma_{3}$. Since $E_{1}$ is globally stable in $\Gamma_{3} \backslash E_{0}$ by Theorem 2, we have $W_{3} \subset W^{S}\left(E_{1}\right)$. Then, we focus on the linearization of system (3) with respect to the subspace $\Gamma_{4}$. Calculating the respective Jacobian matrix $J_{2}$, we obtain

$$
J_{2}=F_{2}-V_{2} \text {, }
$$

where $F_{2}$ and $V_{2}$ are shown in (16). In accordance with Theorem 2 in [14],

$$
s\left(J_{2}\right)<0(>0) \Longleftrightarrow \mathscr{R}_{2}^{1}<1(>1),
$$

in which $s(\cdot)$ represents the maximum real part of the eigenvalues of $J_{2}$.

If $\mathscr{R}_{2}^{1}>1$, that is, $s\left(J_{2}\right)>0$, the boundary equilibrium $E_{1}$ is unstable. If $\mathscr{R}_{2}^{1}<1$, the real parts of the eigenvalues of $J_{2}$ are all negative; therefore, $\Gamma_{4}$ is stable. At the same time, the manifold $W_{2}$ is also stable. Then, we can prove that dim $W^{S}\left(E_{1}\right) \geq \operatorname{dim} W_{3}+\operatorname{dim} W_{2}=8$; therefore, $\operatorname{dim} W^{S}\left(E_{1}\right)=8$, and the equilibrium $E_{1}$ is stable. Finally, when $\mathscr{R}_{1}>1$, the equilibrium $E_{1}$ is locally asymptotically stable for system (3) if $\mathscr{R}_{2}^{1}<1$ but unstable if $\mathscr{R}_{2}^{1}>1$.

The proof method of Theorem 4 can also be applied to the following theorem, which shows how the stability of $E_{2}$ depends on the invasion reproduction number $\mathscr{R}_{1}^{2}$.

Theorem 5. When $\mathscr{R}_{2}>1$, the boundary equilibrium $E_{2}$ is locally asymptotically stable for system (3) if $\mathscr{R}_{1}^{2}<1$ but unstable if $\mathscr{R}_{1}^{2}>1$.

4.3. Coexistence of Two Diseases. We have shown that the boundary equilibrium $E_{1}\left(\right.$ or $\left.E_{2}\right)$ is unstable in the tick-host population where disease 1 (or 2) is already prevalent once the invasion reproduction number $\mathscr{R}_{2}^{1}$ (or $\mathscr{R}_{1}^{2}$ ) is greater than one, which means that disease 2 (or 1 ) is able to invade in the population. From this perspective, the instability of $E_{1}$ and $E_{2}$ may be an advantage to study the coexistence of these two diseases. Here, we present Theorem 6 involving disease persistence.

Theorem 6. If $\mathscr{R}_{1}>1, \mathscr{R}_{2}>1, \mathscr{R}_{2}^{1}>1$, and $\mathscr{R}_{1}^{2}>1$, then system (3) remains uniformly persistent, namely, there exists a constant $\varepsilon>0$ such that every solution $\chi(t) \equiv\left(T^{s}(t)\right.$, $\left.T_{1}^{i}(t), T_{2}^{i}(t), T_{3}^{i}(t), H^{s}(t), H_{1}^{i}(t), H_{2}^{i}(t), H_{3}^{i}(t)\right)$ for system (3) with positive initial value $\chi(0) \equiv\left(T^{s}(0), T_{1}^{i}(0), T_{2}^{i}\right.$ (0), $\left.T_{3}^{i}(0), H^{s}(0), H_{1}^{i}(0), H_{2}^{i}(0), H_{3}^{i}(0)\right)$ satisfies

$$
\liminf _{\mathrm{t} \longrightarrow+\infty}\left(T^{\mathrm{s}}, T_{1}^{\mathrm{i}}, T_{2}^{\mathrm{i}}, T_{3}^{\mathrm{i}}, H^{\mathrm{s}}, H_{1}^{\mathrm{i}}, H_{2}^{\mathrm{i}}, H_{3}^{\mathrm{i}}\right) \geq(\varepsilon, \varepsilon, \varepsilon, \varepsilon, \varepsilon, \varepsilon, \varepsilon, \varepsilon) .
$$


Proof. Define

$$
\begin{aligned}
\mathrm{X}_{0}= & \left\{\left(T^{\mathcal{s}}, T_{1}^{i}, T_{2}^{i}, T_{3}^{i}, H^{s}, H_{1}^{i}, H_{2}^{i}, H_{3}^{i}\right)\right. \\
& \in \Gamma \mid T^{s}>0, T_{1}^{i}>0, T_{2}^{i}>0, T_{3}^{i}>0, H^{s}>0, \\
& \left.H_{1}^{i}>0, H_{2}^{i}>0, H_{3}^{i}>0\right\}, \\
\partial \mathrm{X}_{0}= & \Gamma \backslash \mathrm{X}_{0} .
\end{aligned}
$$

It can be shown that system (3) remains uniformly persistent regarding $\left(\mathrm{X}_{0}, \partial \mathrm{X}_{0}\right)$.

By Lemma 1, first of all, we know that $\Gamma$ and $X_{0}$ are positively invariant in system (3). Also, $\mathrm{X}_{0}$ is relatively closed in $\Gamma$, and system (3) is point dissipative. Let

$M_{\partial}=\left\{\chi(t) \mid \chi(t)\right.$ satisfies system (3) as well as $\left.\chi(t) \in \partial \mathrm{X}_{0}, \forall \mathrm{t} \geq 0\right\}$,

and set

$$
\begin{aligned}
& \bar{M}_{\partial 0}=\left\{\left(T^{s}, 0,0,0, H^{s}, 0,0,0\right) \mid T^{s} \geq 0, H^{s} \geq 0\right\}, \\
& \bar{M}_{\partial 1}=\left\{\left(T^{s}, T_{1}^{i}, 0,0, H^{s}, H_{1}^{i}, 0,0\right) \mid T^{s} \geq 0, H^{s} \geq 0, T_{1}^{i} \geq 0, H_{1}^{i} \geq 0\right\}, \\
& \bar{M}_{\partial 2}=\left\{\left(T^{s}, 0, T_{2}^{i}, 0, H^{s}, 0, H_{2}^{i}, 0\right) \mid T^{s} \geq 0, H^{s} \geq 0, T_{2}^{i} \geq 0, H_{2}^{i} \geq 0\right\} .
\end{aligned}
$$

Assume $\bar{M}_{\partial}=\bar{M}_{\partial 0} \cup \bar{M}_{\partial 1} \cup \bar{M}_{\partial 2}$. Then, we prove that

$$
M_{\partial}=\bar{M}_{\partial} \text {. }
$$

Firstly, $\bar{M}_{\partial} \subseteq M_{\partial}$ clearly holds, so we concentrate on the proof of $M_{\partial} \subseteq \bar{M}_{\partial}$.

Proving by contradiction, we suppose that $M_{\partial} \subseteq \bar{M}_{\partial}$ does not hold. As $\chi(t)$ denotes the solution of system (3) with positive initial value $\chi(0)$, then $\chi(t) \in M_{\partial}$ for $t \geq 0$, and $\chi(t) \notin \bar{M}_{\partial}$. Thus, $\chi(t) \equiv\left(T^{s}(t), T_{1}^{i}(t), T_{2}^{i}(t), T_{3}^{i}(t), H^{s}(t)\right.$, $\left.H_{1}^{i}(t), H_{2}^{i}(t), H_{3}^{i}(t)\right)$ is in accordance with one of these conditions:

(1) There exists $t_{0} \geq 0$ such that at least one of $T_{3}^{i}\left(t_{0}\right)$ and $H_{3}^{i}\left(t_{0}\right)$ is not zero

(2) If $T_{3}^{i}\left(t_{0}\right)=H_{3}^{i}\left(t_{0}\right)=0$ as $t_{0} \geq 0$, then the solution satisfies the following: (i) there exists $t_{0} \geq 0$ such that at least one of $T_{1}^{i}\left(t_{0}\right)$ and $H_{1}^{i}\left(t_{0}\right)$ is not zero; (ii) there exists $t_{0} \geq 0$ such that at least one of $T_{2}^{i}\left(t_{0}\right)$ and $H_{2}^{i}\left(t_{0}\right)$ is not zero

We suppose $\chi(t)$ satisfies condition (1). Without loss of generality, we may as well presume that $T_{3}^{i}\left(t_{0}\right)>0$ and $H_{3}^{i}\left(t_{0}\right)=0$. According to Lemma 1 , the solution $T_{3}^{i}(t)>0$ can be given for any $t>t_{0}$. For system (3) and for $t>t_{0}$, we have

$$
\begin{aligned}
& H_{1}^{i}(t)= H_{1}^{i}\left(t_{0}\right) \exp \left\{-\int_{t_{0}}^{t} \alpha_{12} \frac{T_{2}^{i}(s)}{H} d s-\int_{t_{0}}^{t} \alpha_{13} \frac{T_{3}^{i}(s)}{H} d s-\mu_{H}\left(t-t_{0}\right)\right\} \\
&+\exp \left\{-\int_{t_{0}}^{t} \alpha_{12} \frac{T_{2}^{i}(s)}{H} d s-\int_{t_{0}}^{t} \alpha_{13} \frac{T_{3}^{i}(s)}{H} d s-\mu_{H}\left(t-t_{0}\right)\right\} \int_{t_{0}}^{t}\left[\alpha_{1} T_{1}^{i}(s) \frac{H^{s}(s)}{H}\right. \\
&\left.+\alpha_{31} T_{3}^{i}(s) \frac{H^{s}(s)}{H}\right] \exp \left\{\int_{s}^{t}\left[\alpha_{12} \frac{T_{2}^{i}(\xi)}{H}+\alpha_{13} \frac{T_{3}^{i}(\xi)}{H}\right] d \xi+\mu_{H}(t-s)\right\} d s>0 \\
& H_{2}^{i}(t)= H_{2}^{i}\left(t_{0}\right) \exp \left\{-\int_{t_{0}}^{t} \alpha_{21} \frac{T_{1}^{i}(s)}{H} d s-\int_{t_{0}}^{t} \alpha_{23} \frac{T_{3}^{i}(s)}{H} d s-\mu_{H}\left(t-t_{0}\right)\right\} \\
&+\exp \left\{-\int_{t_{0}}^{t} \alpha_{21} \frac{T_{1}^{i}(s)}{H} d s-\int_{t_{0}}^{t} \alpha_{23} \frac{T_{3}^{i}(s)}{H} d s-\mu_{H}\left(t-t_{0}\right)\right\} \int_{t_{0}}^{t}\left[\alpha_{2} T_{2}^{i}(s) \frac{H^{s}(s)}{H}\right. \\
&\left.+\alpha_{32} T_{3}^{i}(s) \frac{H^{s}(s)}{H}\right] \exp \left\{\int_{s}^{t}\left[\alpha_{21} \frac{T_{1}^{i}(\xi)}{H}+\alpha_{23} \frac{T_{3}^{i}(\xi)}{H}\right] d \xi+\mu_{H}(t-s)\right\} d s>0, \\
&\left.+\beta_{31} H_{3}^{i}(s) \frac{T^{s}(s)}{H}\right] \exp \left\{\int_{s}^{t}\left[\beta_{12} \frac{H_{2}^{i}(\xi)}{H}+\beta_{13} \frac{H_{3}^{i}(\xi)}{H}\right] d \xi+\mu_{T}(t-s)\right\} d s>0 \\
& T_{1}^{i}(t)= T_{1}^{i}\left(t_{0}\right) \exp \left\{-\int_{t_{0}}^{t} \beta_{12} \frac{H_{2}^{i}(s)}{H} d s-\int_{t_{0}}^{t} \beta_{13} \frac{H_{3}^{i}(s)}{H} d s-\mu_{T}\left(t-t_{0}\right)\right\} \\
&\left.\beta_{12} \frac{H_{2}^{i}(s)}{H} d s-\int_{t_{0}}^{t} \frac{\beta_{3}^{i}(s)}{H} d s-\mu_{H}\left(t-t_{0}\right)\right\} \int_{t_{0}}^{t}\left[\beta_{1} H_{1}^{i}(s) \frac{T^{s}(s)}{H}\right.
\end{aligned}
$$




$$
\begin{aligned}
T_{2}^{i}(t)= & T_{2}^{i}\left(t_{0}\right) \exp \left\{-\int_{t_{0}}^{t} \beta_{21} \frac{H_{1}^{i}(s)}{H} d s-\int_{t_{0}}^{t} \beta_{23} \frac{H_{3}^{i}(s)}{H} d s-\mu_{T}\left(t-t_{0}\right)\right\} \\
& +\exp \left\{-\int_{t_{0}}^{t} \beta_{21} \frac{H_{1}^{i}(s)}{H} d s-\int_{t_{0}}^{t} \beta_{23} \frac{H_{3}^{i}(s)}{H} d s-\mu_{T}\left(t-t_{0}\right)\right\} \int_{t_{0}}^{t}\left[\beta_{2} H_{2}^{i}(s) \frac{T^{s}(s)}{H}\right. \\
& \left.+\beta_{32} H_{3}^{i}(s) \frac{T^{s}(s)}{H}\right] \exp \left\{\int_{s}^{t}\left[\beta_{21} \frac{H_{1}^{i}(\xi)}{H}+\beta_{23} \frac{H_{3}^{i}(\xi)}{H}\right] d \xi+\mu_{T}(t-s)\right\} d s>0, \\
H_{3}^{i}(t)= & H_{3}^{i}\left(t_{0}\right) \exp \left\{-\mu_{H}\left(t-t_{0}\right)\right\}+\exp \left\{-\mu_{H}\left(t-t_{0}\right)\right\} \int_{t_{0}}^{t}\left[\alpha_{3} T_{3}^{i}(s) \frac{H^{s}(s)}{H}\right. \\
& \left.+\alpha_{12} T_{2}^{i}(s) \frac{H_{1}^{i}(s)}{H}+\alpha_{13} T_{3}^{i}(s) \frac{H_{1}^{i}(s)}{H}+\alpha_{21} T_{1}^{i}(s) \frac{H_{2}^{i}(s)}{H}+\alpha_{23} T_{3}^{i}(s) \frac{H_{2}^{i}(s)}{H}\right] \\
& \exp \left\{\mu_{H}(t-s)\right\} d s>0 .
\end{aligned}
$$

This implies that $\chi(t) \notin \partial \mathrm{X}_{0}$ when $t>t_{0}$. Likewise, we have $\chi(t) \notin \partial \mathrm{X}_{0}$ for $t>t_{0}$ using condition (2), which contradicts the hypothesis $\chi(t) \in \partial \mathrm{X}_{0}$. Therefore, it follows that $M_{\partial} \subseteq \bar{M}_{\partial}$, and then $M_{\partial}=\bar{M}_{\partial}$.

We know that there exist three equilibria $E_{0}, E_{1}$, and $E_{2}$ in which all are isolated invariant sets in $\Gamma$. In addition, we can show that $W^{S}\left(E_{0}\right) \cap \mathrm{X}_{0}=\varnothing, W^{S}\left(E_{1}\right) \cap \mathrm{X}_{0}=\varnothing$, and $W^{S}\left(E_{2}\right) \cap \mathrm{X}_{0}=\varnothing$, in which $W^{S}\left(E_{i}\right)$ represents the stable manifold of $E_{i}$ for $i=0,1,2$, namely, there is $\kappa>0$ such that, for every solution $\Phi_{t}(\chi(0))$ of system (3) with $\chi(0) \in \mathrm{X}_{0}$, we obtain

$$
\begin{aligned}
& \limsup _{t \longrightarrow+\infty} d\left(\Phi_{t}(\chi(0)), E_{0}\right) \geq \kappa, \\
& \limsup _{t \longrightarrow+\infty} d\left(\Phi_{t}(\chi(0)), E_{1}\right) \geq \kappa, \\
& \limsup _{t \rightarrow+\infty} d\left(\Phi_{t}(\chi(0)), E_{2}\right) \geq \kappa,
\end{aligned}
$$

in which $d$ is a distance function for $\mathrm{X}_{0}$.

Without loss of generality, we only prove that limsup $\sup _{\mathrm{t} \longrightarrow+\infty} d\left(\Phi_{t}(\chi(0)), E_{1}\right) \geq \kappa$. Suppose it does not hold; then, $\lim \sup _{\mathrm{t} \longrightarrow+\infty} d\left(\Phi_{t}(\chi(0)), E_{1}\right)<\varepsilon$ for any $\varepsilon>0$, that is, there is $t_{4}=t(\varepsilon)>0$ such that $T^{s *}-\varepsilon \leq T^{s} \leq T^{s *}+\varepsilon$, $T_{1}^{i *}-\varepsilon \leq T_{1}^{i} \leq T_{1}^{i *}+\varepsilon, H^{s *}-\varepsilon \leq H^{s} \leq H^{s *}+\varepsilon, H_{1}^{i *}-\varepsilon \leq H_{1}^{i}$ $\leq H_{1}^{i *}+\varepsilon, 0 \leq T_{2}^{i} \leq \varepsilon, 0 \leq T_{3}^{i} \leq \varepsilon, 0 \leq H_{2}^{i} \leq \varepsilon$, and $0 \leq H_{3}^{i} \leq \varepsilon$ for any $t>t_{4}$. In system (3) for $t>t_{4}$, we have

$$
\begin{gathered}
\frac{d T_{2}^{i}(t)}{d t} \geq \beta_{2} H_{2}^{i} \frac{T^{s *}-\varepsilon}{H^{*}}+\beta_{32} H_{3}^{i} \frac{T^{s *}-\varepsilon}{H^{*}}-\beta_{21} T_{2}^{i} \frac{H_{1}^{i *}+\varepsilon}{H^{*}}-\beta_{23} \frac{\varepsilon^{2}}{H^{*}}-\mu_{T} T_{2}^{i}, \\
\frac{d T_{3}^{i}(t)}{d t} \geq \beta_{3} H_{3}^{i} \frac{T^{s *}-\varepsilon}{H^{*}}+\left(\beta_{12} H_{2}^{i}+\beta_{13} H_{3}^{i}\right) \frac{T_{1}^{i *}-\varepsilon}{H^{*}}+\beta_{21} T_{2}^{i} \frac{H_{1}^{i *}-\varepsilon}{H^{*}}-\mu_{T} T_{3}^{i}, \\
\frac{d H_{2}^{i}(t)}{d t} \geq \alpha_{2} T_{2}^{i} \frac{H^{s *}-\varepsilon}{H^{*}}+\alpha_{32} T_{3}^{i} \frac{H^{s *}-\varepsilon}{H^{*}}-\alpha_{21} H_{2}^{i} \frac{T_{1}^{i *}+\varepsilon}{H^{*}}-\alpha_{23} \frac{\varepsilon^{2}}{H^{*}}-\mu_{H} H_{2}^{i}, \\
\frac{d H_{3}^{i}(t)}{d t} \geq \alpha_{3} T_{3}^{i} \frac{H^{s *}-\varepsilon}{H^{*}}+\left(\alpha_{12} T_{2}^{i}+\alpha_{13} T_{3}^{i}\right) \frac{H_{1}^{i *}-\varepsilon}{H^{*}}+\alpha_{21} H_{2}^{i} \frac{T_{1}^{i *}-\varepsilon}{H^{*}}-\mu_{H} H_{3}^{i} .
\end{gathered}
$$

Then, we consider the following auxiliary system:

$$
\frac{d u}{d t}=M_{2}(\varepsilon) u
$$

in which the vector $u=\left(u_{1}, u_{2}, u_{3}, u_{4}\right)^{T}$ and 


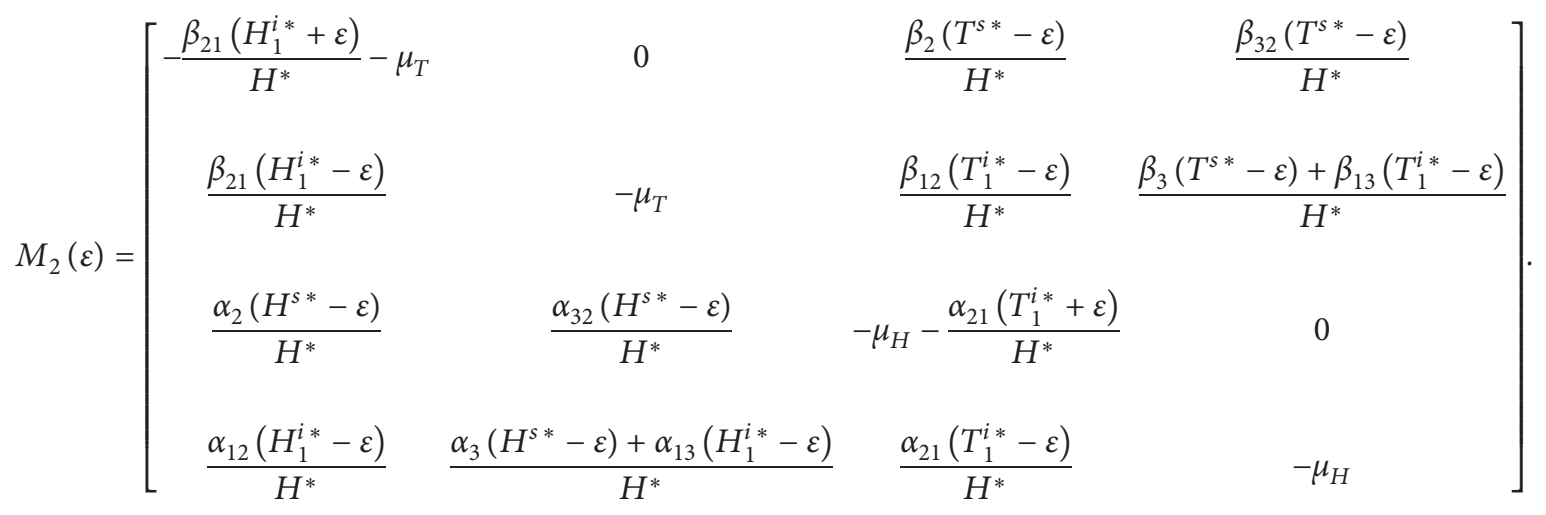

It is clear that $M_{2}(0)=F_{2}-V_{2}$, where $F_{2}$ and $V_{2}$ are given by (16). By referring to Theorem 2 in [14], we get that if $\mathscr{R}_{2}^{1}>1$, then $s\left(M_{2}(0)\right)>0$. As $M_{2}(\varepsilon)$ is continuous for small $\varepsilon$, there exists sufficiently small $\varepsilon$ such that $s\left(M_{2}(\varepsilon)\right)>0$. Consequently, we obtain a positive eigenvalue of $M_{2}(\varepsilon)$ with a positive eigenvector. Let $u(t)=\left(u_{1}(t), u_{2}(t), u_{3}(t)\right.$, $\left.u_{4}(t)\right)^{T}$ denote a positive solution for auxiliary system $(50)$, which is strictly increasing, namely, $u_{i}(t) \longrightarrow+\infty$ as $t \longrightarrow+\infty$ for $i=1,2,3,4$. Using the comparison principle, we have

$$
\begin{aligned}
& \lim _{t \longrightarrow+\infty} T_{2}^{i}(t)=+\infty, \lim _{t \longrightarrow+\infty} T_{3}^{i}(t)=+\infty, \\
& \lim _{t \longrightarrow+\infty} H_{2}^{i}(t)=+\infty, \lim _{t \longrightarrow+\infty} H_{3}^{i}(t)=+\infty,
\end{aligned}
$$

and this conflicts with the above assumption.

Thus, $\left\{E_{1}\right\}$ is not only an isolated invariant set for $\Gamma$, but it also satisfies $W^{S}\left(E_{1}\right) \cap \mathrm{X}_{0}=\varnothing$. Analogously, we can also verify that $\left\{E_{2}\right\}$ is not only an isolated invariant set for $\Gamma$, but it also satisfies $W^{S}\left(E_{2}\right) \cap \mathrm{X}_{0}=\varnothing$ when $\mathscr{R}_{1}^{2}>1$. In addition, when $\mathscr{R}_{1}>1$ and $\mathscr{R}_{2}>1,\left\{E_{0}\right\}$ is not only an isolated invariant set for $\Gamma$, but it also satisfies $W^{S}\left(E_{0}\right) \cap \mathrm{X}_{0}=\varnothing$. According to Thieme [20], system (3) is uniformly persistent if $\mathscr{R}_{1}>1, \mathscr{R}_{2}>1, \mathscr{R}_{2}^{1}>1$, and $\mathscr{R}_{1}^{2}>1$.

\section{Numerical Illustrations and Discussion}

In this section, we utilize specific numerical simulations to illustrate the impact of coinfection on the dynamics of two tick-borne pathogens and ensure the effectiveness of the previous theories. Besides, we also propose and verify the uniform persistence of the two diseases and the presence of a coexistence equilibrium in the case in which $\mathscr{R}_{1}>1$ and $\mathscr{R}_{2}<1<\mathscr{R}_{2}^{1}$ or $\mathscr{R}_{2}>1$ and $\mathscr{R}_{1}<1<\mathscr{R}_{1}^{2}$ in the following.

By referring to some early works [2,11, 21-23], we fix the parameter values as follows: $\Lambda_{T}=5000 \mathrm{day}^{-1}, \mu_{T}=1 \mathrm{day}^{-1}$, $\Lambda_{H}=200$ day $^{-1}, \mu_{H}=0.1$ day $^{-1}, \beta_{1}=0.4, \alpha_{1}=0.3, \beta_{2}=$ 0.25 , and $\alpha_{2}=0.15$. First, we consider subsystems (33) and (37) and set the initial values of these subsystems to $\left(T^{s}\right.$ $\left.(0), T_{1}^{i}(0), H^{s}(0), H_{1}^{i}(0)\right)=\left(T^{s}(0), T_{2}^{i}(0), H^{s}(0), H_{2}^{i}(0)\right)=$ $(100,250,75,200)$. According to Figure 2(a), it is clearly shown that the number of infectious individuals with disease 1 consisting of infected ticks and hosts will eventually reach the equilibrium $E_{1}^{1}=(4105,952.5,853.5,1146)$, which means that disease 1 would remain endemic since $\mathscr{R}_{1}=1.025>1$. On the contrary, it follows from Figure 2(b) that disease 2 will not persist in the relative tick and host populations as expected since $\mathscr{R}_{2}=0.943<1$. Thus, we can conclude that disease 2 cannot survive alone in tick and host populations, but disease 1 can.

Next, we introduce disease 2, which cannot spread if taken singularly, into tick and host populations where disease 1 already exists in order to study the influence of coinfection. We choose the following as parameter values: $\beta_{32}=\beta_{31}=\beta_{21}=$ $\beta_{23}=\alpha_{32}=\alpha_{31}=\alpha_{23}=\alpha_{21}=0.1, \beta_{3}=\alpha_{3}=0.25$, and $\beta_{12}=$ $\beta_{13}=\alpha_{12}=\alpha_{13}=0.12$. The initial values of $\left(T_{2}^{i}, T_{3}^{i}, H_{2}^{i}, H_{3}^{i}\right)$ are set to $\left(T_{2}^{i}(0), T_{3}^{i}(0), H_{2}^{i}(0), H_{3}^{i}(0)\right)=(100,200$, $150,100)$, and we calculate the invasion reproduction number $\mathscr{R}_{2}^{1}=1.01>1$. From Figure 3, we see that infected ticks and hosts with only disease 2 approach and stabilize at 76.4 and 65.9, and ones with both pathogens stabilize at 153.7 and 302.9, respectively, which imply that disease 2 can invade tick and host populations in which disease 1 is already endemic if $\mathscr{R}_{2}^{1}>1$. In order to visualize theoretical results, we choose $\beta_{3}$ and $\alpha_{3}$ as parameters and plot Figure 4 to show their effects on the invasion reproduction number $\mathscr{R}_{2}^{1}$.

In this paper, we have developed a coinfection model involving two tick-borne diseases to explore their interaction and spread. Based primarily on the works by Van den Driessche [14], we have calculated the basic reproduction numbers concerning the disease-free equilibrium $E_{0}$ and two boundary equilibria $E_{1}$ and $E_{2}$. Considering the invasion of a disease, we have then proposed a new threshold, namely, the invasion reproduction number, to describe the number of secondary cases infected by a disease later introduced into a population where another disease is already endemic, and we have derived the expressions for these two reproduction numbers.

According to the comparison principle and the theory of asymptotically semiflow, we have proved the global stability at the disease-free equilibrium $E_{0}$ if $\mathscr{R}_{0}<1$ and the local stability at the two boundary equilibria $E_{1}$ and $E_{2}$ if $\mathscr{R}_{1}>1\left(\mathscr{R}_{2}>1\right)$ and $\mathscr{R}_{2}^{1}<1\left(\mathscr{R}_{1}^{2}<1\right)$, as well as the coexistence conditions for these two diseases, which are $\mathscr{R}_{1}>1, \mathscr{R}_{2}>1, \mathscr{R}_{2}^{1}>1$, and $\mathscr{R}_{1}^{2}>1$. We have also showed that the two diseases are uniformly persistent, and the transmission system of two diseases admits a coexistence equilibrium when $\mathscr{R}_{1}>1$ (or $\mathscr{R}_{2}>1$ ) and $\mathscr{R}_{2}<1<\mathscr{R}_{2}^{1}$ (or 


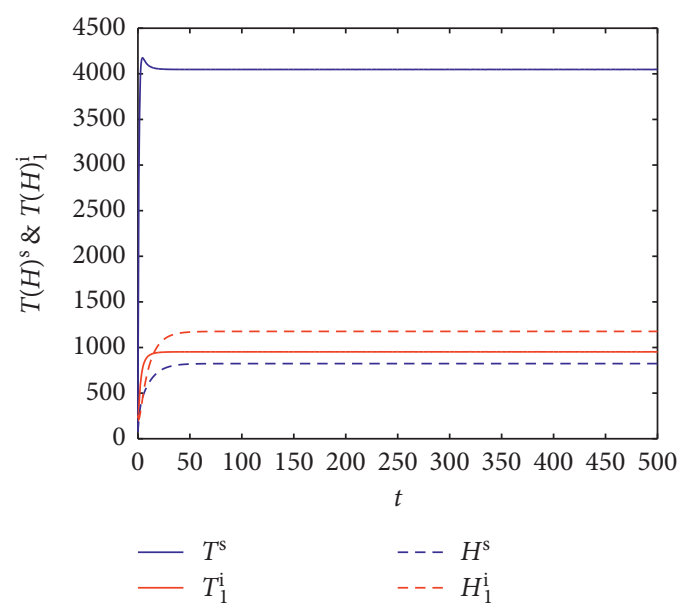

(a)

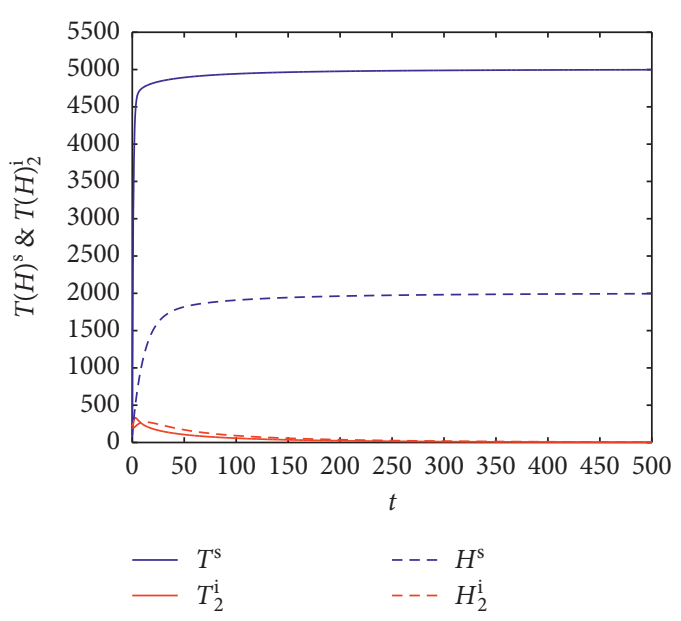

(b)

Figure 2: Solutions of submodels (33) and (37). The numbers of susceptible and infectious individuals are represented by blue and red curves, respectively, where solid lines denote the number of susceptible or infectious ticks, and dotted lines denote the number of susceptible or infectious hosts. (a) Subsystem (33) stabilizes at equilibrium $E_{1}^{1}$ as $\mathscr{R}_{1}=1.025>1$ for this parameter choice, which indicates that disease 1 can be endemic in tick and host populations; (b) susceptible ticks and hosts eventually approach and stabilize at 4990 and 1985, respectively, but infected ticks and hosts die out as $\mathscr{R}_{2}=0.943<1$, which implies that disease 2 cannot spread in the tick-host population.

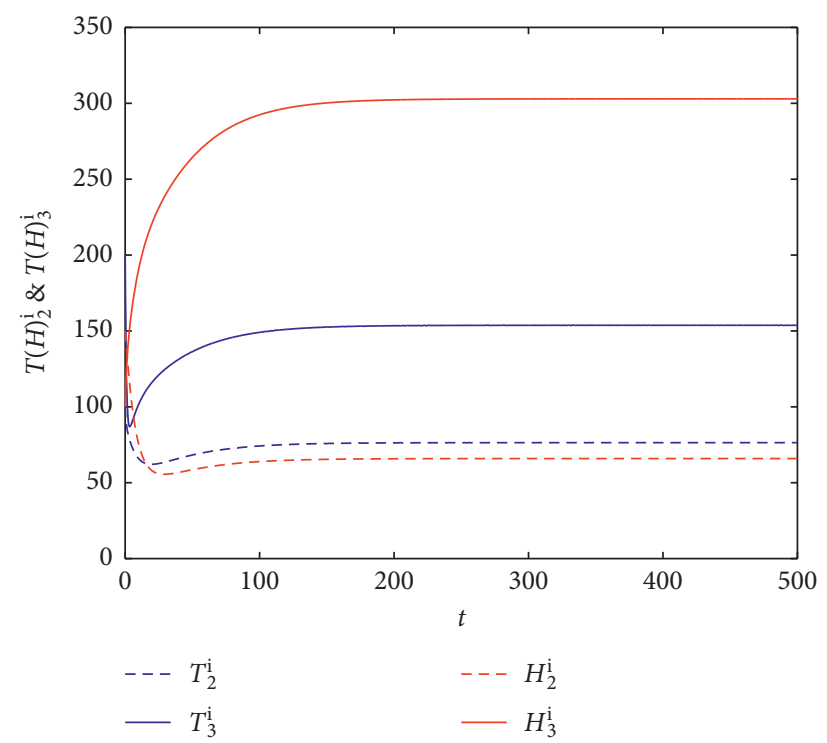

Figure 3: Solutions of model (3) reflecting the influence of the invasion of disease 2. The numbers of infectious ticks and hosts are represented by blue and red curves, respectively, where dotted lines denote the number of infected individuals with only disease 2 , and solid lines denote the number of infected individuals with both diseases. It is shown that infectious individuals related to both diseases tend to persist, and then disease 2 can invade the tick-host population as $\mathscr{R}_{2}^{1}=1.01>1$.

$\mathscr{R}_{1}<1<\mathscr{R}_{1}^{2}$ ), which implies that the existence of one disease can facilitate the invasion and transmission of another disease. These results have been verified by numerical simulations.

The highlight of this paper is the introduction of the invasion reproduction number, which can directly reflect whether one disease can invade a population where another disease is already persistent, in order to investigate the effect of coinfection. We conclude that coinfection has a positive effect on the transmission of two tick-borne diseases in model (3), that is, one disease may invade a tick-host population where another disease already exists, although it cannot remain endemic alone in the population.

However, theoretically, pathogens or diseases interacting in a population may not only promote each other but also compete or be independent one from the other [2]. Thus, in future research, we will focus on the negative effects of coinfection on diseases transmission. Furthermore, other factors such as temporal and spatial heterogeneity will also be worth discussing. 


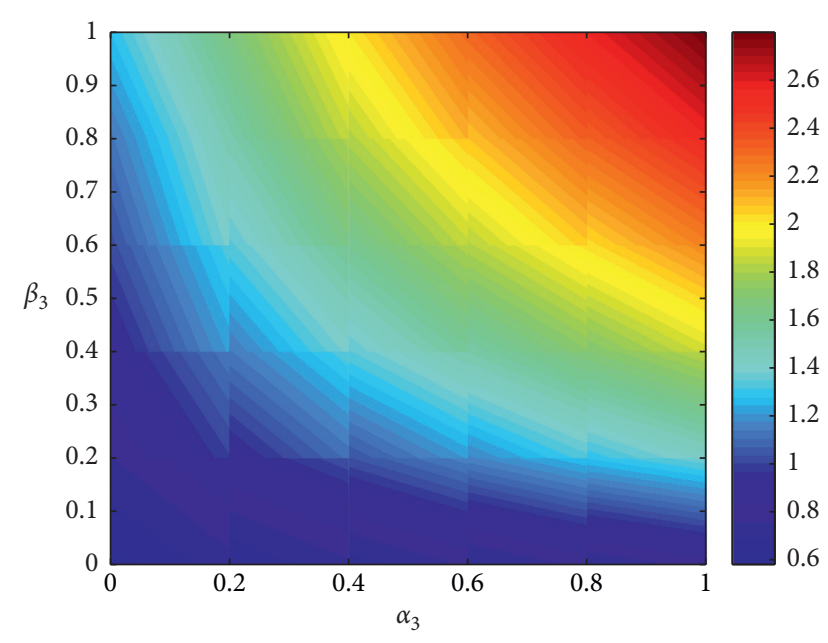

FIGURE 4: Effects of $\beta_{3}$ and $\alpha_{3}$ on the invasion reproduction number $\mathscr{R}_{2}^{1}$.

\section{Data Availability}

The data used to support the findings of this study are included within the article.

\section{Conflicts of Interest}

The authors declare that they have no conflicts of interest.

\section{Acknowledgments}

This work was supported by the Natural Science Foundation of China (no. 11701072).

\section{References}

[1] Center for Disease Control and Prevention (CDC), "Lyme disease-United States," MMWR-Morbidity and Mortality Weekly Report, vol. 56, no. 23, pp. 573-576, 2007.

[2] Y. Lou, L. Liu, and D. Gao, "Modeling co-infection of Ixodes tick-borne pathogens," Mathematical Biosciences and Engineering, vol. 14, no. 5, pp. 1301-1316, 2017.

[3] M. H. Hersh, R. S. Ostfeld, D. J. McHenry et al., "Co-infection of blacklegged ticks with Babesia microti and Borrelia burgdorferi is higher than expected and acquired from small mammal hosts," PloS one, vol. 9, no. 6, 2014.

[4] L. Halos, T. Jamal, R. Maillard et al., "Evidence of Bartonella $s p$. in questing adult and nymphal Ixodes ricinus ticks from France and co-infection with Borrelia burgdorferi sensu lato and Babesia sp." Veterinary Research, vol. 36, no. 1, pp. 79-87, 2005.

[5] S. J. Swanson, D. Neitzel, K. D. Reed, and E. A. Belongia, "Coinfections acquired from Ixodes ticks," Clinical Microbiology Reviews, vol. 19, no. 4, pp. 708-727, 2006.

[6] M. A. Diuk-Wasser, E. Vannier, and P. J. Krause, "Coinfection by Ixodes tick-borne pathogens: ecological, epidemiological, and clinical consequences," Trends in Parasitology, vol. 32, no. 1, pp. 30-42, 2016.

[7] H. W. Horowitz, M. E. Aguero-Rosenfeld, D. Holmgren et al., "Lyme disease and human granulocytic anaplasmosis coinfection: impact of case definition on coinfection rates and illness severity," Clinical Infectious Diseases, vol. 56, no. 1, pp. 93-99, 2013.

[8] R. Welc-Faleciak, A. Hildebrandt, and E. Sinski, "Co-infection with Borrelia species and other tick-borne pathogens in humans: two cases from Poland," Annals of Agricultural and Environmental Medicine, vol. 17, pp. 309-313, 2010.

[9] D. Gao, T. C. Porco, and S. Ruan, "Coinfection dynamics of two diseases in a single host population," Journal of Mathematical Analysis and Applications, vol. 442, no. 1, pp. 171-188, 2016.

[10] B. Tang, Y. Xiao, and J. Wu, "Implication of vaccination against dengue for Zika outbreak," Scientific Reports, vol. 6, no. 1, p. 35623, 2016.

[11] L. Wang and H. Zhao, "Dynamics analysis of a Zika-dengue co-infection model with dengue vaccine and antibody-dependent enhancement," Physica A: Statistical Mechanics and Its Applications, vol. 522, pp. 248-273, 2019.

[12] J. Heffernan, Y. Lou, and J. Wu, "Range expansion of Ixodes scapularis ticks and of Borrelia burgdorferi by migratory birds," Discrete and Continuous Dynamical Systems-Series B, vol. 19, no. 10, pp. 3147-3167, 2014.

[13] R. Rosà, A. Pugliese, R. Norman, and P. J. Hudson, "Thresholds for disease persistence in models for tick-borne infections including non-viraemic transmission, extended feeding and tick aggregation," Journal of Theoretical Biology, vol. 224, no. 3, pp. 359-376, 2003.

[14] P. Van den Driessche and J. Watmough, "Reproduction numbers and sub-threshold endemic equilibria for compartmental models of disease transmission," Mathematical Biosciences, vol. 180, no. 1-2, pp. 29-48, 2002.

[15] Z. Feng, Z. Qiu, Z. Sang, C. Lorenzo, and J. Glasser, "Modeling the synergy between HSV-2 and HIV and potential impact of HSV-2 therapy," Mathematical Biosciences, vol. 245, no. 2, pp. 171-187, 2013.

[16] Y. Lou and X.-Q. Zhao, "A climate-based malaria transmission model with structured vector population," SIAM Journal on Applied Mathematics, vol. 70, no. 6, pp. 2023-2044, 2010.

[17] X.-Q. Zhao, Dynamical Systems in Population Biology, Springer-Verlag, Berlin, Germany, 2003.

[18] Y. Lou and X.-Q. Zhao, "The periodic Ross-MacDonald model with diffusion and advection," Applicable Analysis, vol. 89, no. 7, pp. 1067-1089, 2010.

[19] D. Gao, Y. Lou, and S. Ruan, "A periodic Ross-MacDonald model in a patchy environment," Discrete and Continuous Dynamical Systems-Series B, vol. 19, no. 10, pp. 3133-3145, 2014.

[20] H. R. Thieme, "Persistence under relaxed point-dissipativity (with application to an endemic model)," SIAM Journal on Mathematical Analysis, vol. 24, no. 2, pp. 407-435, 1993.

[21] R. Rosà and A. Pugliese, "Effects of tick population dynamics and host densities on the persistence of tick-borne infections," Mathematical Biosciences, vol. 208, no. 1, pp. 216-240, 2007.

[22] N. A. Hartemink, S. E. Randolph, S. A. Davis, and J. A. P. Heesterbeek, "The basic reproduction number for complex disease systems: defining $R_{0}$ for tick-borne infections," The American Naturalist, vol. 171, no. 6, pp. 743-754, 2008.

[23] X. Zhang, X. Wu, and J. Wu, "Critical contact rate for vectorhost-pathogen oscillation involving co-feeding and diapause," Journal of Biological Systems, vol. 25, no. 4, pp. 657-675, 2017. 